sgrammaticata e mimeticamente frettolosa della popolaresca caduta della proposizione in "Se dalle tue lunghe agonie e dai miei brevi respiri/sorgesse un fiore; allora io correrei ringraziarti/rimboccherei la strada della bellezza. [...]», «Si de tus largas agonías y mis cortos suspiros/surgiera una flor; entonces correría a agradecerte/enhebrando la calle de tu belleza [...]», dove il gerundio dello spagnolo potrebbe, che so, anche giocare con la similarità del significante tra rimboccare e re-imboccare, ma non è facile: il trobar clus, spesso bifido, beffardo, di Rosselli se non lesina irrisioni e trappole ai propri stessi pronunciamenti gnomici, molte ne riserva anche a chi vi si accosti, insieme invitandolo ad una copula illogica, e respingendolo. Ed è spesso vivace Merlo nel reagire, tra fedeltà alle evidenze strutturanti più marcate (non tocca quasi le rumorose anafore che rinserrano i testi) e reinvenzione ritmica, quando l'urgenza traboccante ma avvitata del martellamento sonoro, percussivo, viene risaltata dall'iconismo metrico-sintattico nel testo tradotto, mediante i frequenti encabalgamientos inventati ex nibilo o resi più affilati e violenti rispetto alle inarcature, per tradizione leni, dell'italiano.

Rosselli, con un suo estremo testo critico tradotto è la vestale incaricata di presentare la raccolta Residenze Invernali dell'ottima Anedda, sempre per Igitur, nella versione dell'esperto Emilio Coco, responsabile di utili repertori in castigliano della lirica italiano tardonovecentesca. A Coco si deve pure il sobrio omaggio in postfazione a questo lirico nuovo. Il tutto è davvero squisito e rarefatto e sul resto spicca senz'altro l'eccellente aderenza alla discrezione pudica della voce dell'originale, la morbida fermezza a non cedere alla tentazione di alzare i decibel di una voce sommessa ed elegante (quanto Montale e quanto Luzi, ma nascosti, introflessi), che corteggia il silenzio, con mormorata sacralità achmatoviana. Forse con più acume percettivo si sarebbero potuti rendere quei lessemi di senso che sottilmente nei testi di Anedda trafiggono il silenzio o punteggiano la penombra. «Le nostre anime dovrebbero dormire/come dormono i corpi sottili/stare tra le lenzuola come un foglio/i capelli dietro le orecchie aperte/capaci di ascoltare. Carne/appuntita e fragile, cava/nel buio della stanza». Per un lirico che si pronuncia così è importante che lo scalpiccio sia tale, e così il fruscio, il tonfo, lo scintillio.

Piero Dal Bon

\title{
Eugenio MONTALE
}

\section{Las ocasiones}

Trad. de Carlos Vitale. Montblanc: Igitur/poesía, 2005

Le occasioni di Eugenio Montale finalmente godono di una traduzione allo spagnolo in volume autonomo ed esaustiva riguardo al corpus testuale. Il lavoro, merito dell'argentino Carlos Vitale, costituisce una resa fedele, letterale dei testi, che rispetta il formato dell'originale fino ai minimi dettagli e alla concinnità dello stile che lo definiscono (aspetti tipografici come il corsivo della poesia prelimina- re, Il balcone, la pagina in bianco che separa quest'ultima dal resto del volume e quella che separa i titoli delle singoli parti dal rispettivo corpus costituente; l'esatta disposizione sulla pagina, ecc.); vi sono inoltre le Note d'autore montaliane, tradotte ed inserite in calce al volume come nell'edizione italiana, mentre in apertura vi è un Prólogo di Alfonso Alegre Heitzmann, che tenta una breve ma attenta ed 
approfondita sintesi della poetica montaliana nel suo insieme.

L'edizione che ci propone Igitur/poesía è bilingue, e invece della variante a fronte - testo tradotto ed originale- colloca il secondo a piè di pagina, sicché la traduzione gode di un relativo ma pur presente senso di autonomia sulla pagina.

Non è la prima volta che Carlos Vitale lavora su testi poetici italiani. Poeta lui stesso ed anche narratore, conoscitore della poesia italiana del Novecento, è fra i suoi traduttori più assidui: ha già reso allo spagnolo versi di Campana, di Ungaretti, di Corazzini e di Saba, di Cardarelli, Penna e Zanzotto, nonché di vari autori più recenti, ottenendo numerosi premi italiani, tra cui il premio di traduzione Eugenio Montale, nell'anno 2004. Per Las ocasiones gli è stato assegnato il premio di traduzione Ángel Crespo 2006.

Nell'ambito delle traduzioni in spagnolo de Le occasioni, il lavoro di Vitale va preceduto solo da una versione apparsa anni addietro dell'argentino Horacio Armani (1978), che però, oltre ad essere selettiva riguardo ai testi del corpus italiano, fu pubblicata in edizione comune con gli Ossi di seppia; non ne offre quindi una variante autonoma, come neppure la recentissima traduzione del messicano Fabio Morabito (2006), posteriore al libro vitaliano, che include la raccolta in un'edizione spagnola (la prima, del resto) della poesia completa di Montale. In questo senso, la traduzione di Vitale è veramente la prima autonoma ed esaustiva in spagnolo della seconda raccolta de poeta ligure, ed è inoltre la prima che trova pubblicazione qui, in Spagna.

Oltre a possibili spiegazioni di canone che riguardano la ricezione della poesia montaliana in ambito generalmento spagnolo, le ragioni di questa mancata considerazione delle Occasioni nella loro integrità contenutistica sarebbero da ricercare anche nella stessa materia poetica ivi contenuta, nella sua fattispecie stilistica e testuale. A differenza di quanto avviene negli Ossi di seppia, qui, nelle Occasioni, nell'intenzione auctoris viene meno la volontà estroversa, il bisogno di un contenuto che sia in qualche modo socievole - necessario quanto necessitato nella sua storica urgenza espressiva—, e al luogo dell'esplosione formale e contenutistica vi è l'«implosione»: una specie di «espressionismo capovolto all'interno", per parole di Mengaldo, che spinge il discorso verso una radicale interiorizzazione del linguaggio, specie nel riferente alla sua motivazione deittica. Poesia che prende sostanza con l'occasione, perciò di indole carsica, materia estesa fra i limiti -illimitati- della memoria "che renderà quasi inaccessibile, certo enigmatico, reticente il rapporto con la realtà̀ (N. Lorenzini). Essa è pertanto poco prevedibile nel complesso della sua logica istitutiva: succede, in effetti, con l'avvenire occasionale di un ricordo - contenuto vissutoinsieme all'espressione esatta che ne articola e ne storicizza in situ la validità prospettica e di paradigma. Fors'è precisamente la veste occasionale di questi testi - notule di una autoesegesi-, e al contempo il senso compiuto che soggiace alla loro articolazione formale, a consentirne una lettura isolata e ad ammettere un atteggiamento altrettanto "fortuito", in senso operativo del termine, allorquando vengano concepiti come materia di traduzione. Perciò il lavoro di Vitale, considerandoli viceversa nell'originale formato di raccolta, offre la possibilità di accedere linguisticamente alla realtà di un modello espressivo - un modello di continuità, stilistica e testuale, basato invece sul discontinuo e l'occasionale come agenti strutturali del discorso-, e ravvisarne i principi nonché le pretese di paradigma poetico.

Va ripetuto che si tratta di una resa letterale dei testi che, in linea di fondo, costituisce una valida versione linguistica del corpus al castigliano («Molti anni, e uno più duro sopra il lago / straniero su cui ardono i tramonti. [...] Imprimerli 
potessi sul pavese / che s'agita alla frusta del grecale / in cuore...» : "Muchos años, y uno más duro sobre el lago / extranjero donde arden los ocasos. [...] / Si pudiera imprimirlos en el pavés / que se agita bajo el flagelo del gregal / en el corazón...»: di esempi come questo ce ne sono molti all'interno del volume, essendo la tipologia operativa di Vitale praticamente uniforme ovunque). Ciononostante, qua e là nella traduzione si osserva, da una parte, certa disattenzione nei confronti della prosodia interna dei versi - articolazioni ritmiche, tramandi fonici tra le parole, snodature modali degli accentiche l'Autore ha voluto dotare di forte carico semantico (nel decimo mottetto, Perché tardi?, ad esempio: «Perché tardi? Nel pino lo scoiattolo / batte la coda a torcia sulla scorza. / La mezzaluna scende col suo picco / nel sole che la smorza. È giorno fatto.», dove i tramandi fonici tra alcuni vocaboli reggono la struttura morfosintattica dei versi ed hanno un'evidente missione semantica: la lunga, e lenta, «a» di «tArdi » perde progressivamente importanza deittica a partire dalla «i» di «pino» e per forza dei vocaboli successivi in cui domina l'effetto accorciante e perfettivo delle consonanti doppie-«scoiattolo»-«batte»-«picco»«fatto», e i quali vanno uno dietro l'altro in successione rapida, appoggiati poi dalla rima di «torcia»-«scorza»-«smorza»; di conseguenza, si ottiene un effetto di scontro nozionale tra il «tardare» rivolto al «tu», e cioè il ritmo lento dell'attesa-aspettativa irrisolta che rimane inchiodato nella prima frase interrogativa, e il resto oggettuale del contenuto strofico che invece si risolve, si esaurisce con un ritmo accelerato e pragmatico. Nella versione spagnola, pur non priva di qualche corrispondenza fonica, come quella della «i» di «pino»-«ardilla»- «pico»-«amortigua»-«día», la prosodia scorre secondo un ritmo senza contrasti, piuttosto uniforme $o$ addirittura lento e riflessivo: «¿Por qué tardas? En el pino la ardilla / pega con su cola de antorcha en la corteza. / La media luna desciende con su pico / en el sol que la amortigua. Es pleno día»). Talora si riscontra la presenza di imprecisazioni o sviste lessicali e semantiche (come questa nel mottetto de La rana, dove l'espressione italiana «ritentar[e] la corda" in: "La rana, prima a ritentar la corda / dallo stagno che affossa / giunchi e nubi; [...]», significa «riavviare il canto», e dove nel v.2 la preposizione montaliana non è «di», come intesa da Vitale, bensí «da», per cui «dallo stagno» significa «là dov'è lo stagno»; la variante spagnola appare priva di quest'ultima distinzione semantica e, di conseguenza, si perde il senso contenuto anche nel verso che la precede: «La rana, primera en templar la cuerda / del estanque que ahonda / juncos y nubes; [...]»); altre volte ancora, un cambio nell'ordine della punteggiatura comporta la scorretta interpretazione dell'enunciato montaliano, come nei vv.9-10 di Keepsake: «Falsi spagnoli giocano al castello / i Briganti; [...]», tradotto così: «Falsos españoles, juegan el alcázar / los bandidos; [...]». Tutto ciò si deve forse non tanto a fattori di incompetenza linguistica od operativa, quanto piuttosto ad un'eccessiva fiducia nell'oggettività lessicale del corpus italiano - concreto e schietto nelle raffigurazioni, così puntuale ed unico, in senso di insostituibile, nella materialità del testo, da far considerare ogni vocabolo in sé e per sé, e menomare invece il fatto della sua missione deittica nell'universo testuale di cui fa parte. Si tratta ad ogni qual modo di piccole sbavature isolate che non inficiano l'opera nel suo insieme. Perché la traduzione di Vitale è comunque fruibile e convincente, e denota un accurato lavoro intellettivo. Fra i tanti esempi che ci sono ad avvalorarlo, vi è la resa del montaliano Cave d'autunno, della prima parte della raccolta, ch'è a nostro avviso fra le più più felici dal punto di vista morfosintattico e generalmente stilistico: 
«Cave d'autunno su cui discende la primavera lunare / e nimba di candore ogni frastaglio, / schianti di pigne, abbaglio / di reti stese e schegge, ritornerà ritornerà sul gelo / la bontà di una mano, / varcherà il cielo lontano / la ciurma luminosa che ci saccheggia.»

"Canteras de otoño sobre las que desciende la primavera lunar / y nimba de candor cada contorno, / estallidos de piñas, fulgor / de redes extendidas y de astillas,

retornará, retornará al hielo / la bondad de una mano, atraversará el cielo lejano / la chusma luminosa que nos saquea.»

Forse è il luogo di osservare, che fra le peculiarità stilistiche che definiscono $\mathrm{i}$ versi occasionali di Montale c'è l'implicito, e accovonante, dato di una volontà narrativa che, dal punto di vista della singola struttura dei testi, specie quella dei mottetti e dei testi brevi, appare destinata a realizzarsi a "seconda istanza», cioè di seguito a un incipit al contrario inidentificabile, impersonale. Come se la ragione vera e propria del testo ricuperasse il suo personale contenuto, promossa com'è dalla capacità suggestiva di una situazione d'oggetto. In questo modo, l'enunciato che veramente contiene $\mathrm{i}$ dati di una presenza soggettiva avviene in secondo termine, dopo un'iniziale conquista di spazio testuale ed immaginario. Nel componimento in esame, il fatto da rilevare è che il passaggio dall'indefinito al personale, dalla prima strofe alla seconda, suc- cede in maniera non brusca ma cadenzata, misurata, sicché la valenza soggettiva emerge quale soluzione di continuità semantica di quella oggettiva. Così anche nella variante di Vitale, dove il ricordo di un passato comune, della «bondad de una mano", riemerge in mezzo a "estallidos de piñas, fulgor / de redes extendidas y de astillas». Qui il traduttore riesce a trasmettere linguisticamente il proposito fondamentale dell'Autore, e cioè l'aspirazione a promuovere il contenuto testuale per mezzo della capacità prosodica dei suoi elementi strutturali. Come nell'originale, i singoli vocaboli si ricercano fonicamente e ritmicamente nel traslato spagnolo in virtù di un imperativo semantico: per esempio, nella seconda strofe, dove la forma futura di un verbo - «retornará»_ indica aspirazione e desiderio e perciò richiama fonicamente "la bondad"; "[la bondad]de una mano» che, a sua volta, manca, non c'è, per cui rima con «lejano» del v.6; oppure nell'ultimo verso, in cui il verbo "saquea[r]», oltre a rimare con "cielo» del v.6, richiama (o almeno ricorda) semanticamente lo "hielo» del v.5, ecc. La decisione, poi, di Vitale di volgere al plurale il "cave» montaliano del primo verso, che diventa "canteras", è molto opportuna, perché così viene facilitata l'articolazione dei vocaboli che susseguono e l'enunciato spagnolo, senza perdere di vista l'imperniatura dell'originale, appare dotato di autonomia stilistica ed espressiva.

Elitza Popova 